\title{
Modeling the Effects of Intravasal Administration of AstraZeneca ChAdOx1 nCoV-19 Vaccine on Human Platelets
}

\author{
Pernille Just Vinholt ${ }^{1,2}$ Henrik Frederiksen ${ }^{2,3} \quad$ Christian Nielsen $^{4}$
}

${ }^{1}$ Department of Clinical Biochemistry, Odense University Hospital, Odense, Denmark

${ }^{2}$ Department of Clinical Research, University of Southern Denmark, Denmark

${ }^{3}$ Department of Haematology, Odense University Hospital, Odense, Denmark

${ }^{4}$ Department of Clinical Immunology, Odense University Hospital, Odense, Denmark

Thromb Haemost 2021;121:1681-1683.

The recently introduced ChAdOx1 nCoV-19 (AZD1222) vaccine from Oxford-AstraZeneca can introduce a rare but potentially fatal condition of antibody-mediated vaccineinduced thrombotic thrombocytopenia (VITT), associated with venous thromboembolism and/or bleeding. ${ }^{1,2}$ Affected patients developed spontaneous platelet aggregation, and the majority had high levels of platelet-activating immunoglobulin G (IgG) antibodies against platelet factor 4 (PF4; CXCL4). ${ }^{1,2}$ The phenomenon is observed after a minimum of 5 days following vaccination and the condition is likely caused by autoantibodies produced after the vaccine administration. ${ }^{3}$ However, it has been hypothesized that the pathogenesis may include direct activation of platelets by accidental intravasal administration of this adenovirus vector-based vaccine. This hypothesis is based on the finding that adenovirus binds platelets and causes platelet activation in vitro. ${ }^{3}$ Further, novel data suggest that intravascular administration of adenovirus in animal models causes platelet activation resulting in splenic clearance of aggregated platelets and results in thrombocytopenia. ${ }^{4}$

Our aim was to investigate whether the ChAdOx $1 \mathrm{nCoV}$ 19 vaccine from Oxford-AstraZeneca can directly affect platelet function or cause binding of antibodies to platelets.

For the experiments, the vaccine was added to blood in vitro in a concentration corresponding to a full vaccine dose of $0.5 \mathrm{~mL}$ introduced into the bloodstream. A full description of methods is available in the - Supplementary Material and previously explained in detail. ${ }^{5-7}$ In brief, we evaluated hematology parameters and anti-COV-2IgGII status; platelet activation (percentage of platelets positive for CD62p or
Address for correspondence Pernille Just Vinholt, MD, Department of Clinical Biochemistry and Pharmacology, J. B. Winsløwsvej 4B, Odense University Hospital, Odense, Denmark (e-mail: pernille.vinholt@rsyd.dk).

CD63 and procaspase activating compound-1 [PAC-1] binding) was determined in whole blood using flow cytometry. Further, platelet aggregation response was determined in whole blood (Multiplate, Roche Diagnostics, Mannheim, Germany) and with isolated platelet flow cytometrically. Both the response following stimulation of platelets (with agonists) and the spontaneous response (without agonists) were investigated. Further, global hemostasis was evaluated using the platelet function analyzer (PFA)-200 (Siemens Healthcare, Marburg, Germany). Finally, we tested for drug-induced thrombocytopenia by evaluating IgG binding to intact platelets in the presence of vaccine and/or human PF4 (ChromaTec, Greifswald, Germany) as observed in patients with VITT. ${ }^{2,6}$ Comparisons between samples with and without vaccine were made with paired $t$ test. A $p$-value $<0.05$ was considered significant.

We included six healthy individuals without prior COVID19 disease or vaccination (three men and three women, mean age: 46 years; range: 32-60 years). All individuals were negative for anti-COV-2IgGII and had platelet count within laboratory reference interval. The ChAdOx $1 \mathrm{nCoV}-19$ vaccine from Oxford-AstraZeneca did not cause spontaneous platelet aggregation neither in whole blood using Multiplate nor in isolated platelets using the flow cytometric platelet aggregation assay (-Table $\mathbf{1}$ ). By contrast, there was a statistically significantly lower spontaneous aggregation response with Multiplate in samples with vaccine. We found no difference between platelet activation capacity in terms of platelet granule secretion (percentage of platelets positive for CD62p or CD63 reflecting secretion of platelet $\alpha$ and received

July 26, 2021

accepted

August 3, 2021

published online

August 5, 2021 (c) 2021. Thieme. All rights reserved. Georg Thieme Verlag KG,

Rüdigerstraße 14,

70469 Stuttgart, Germany
DOI https://doi.org/ 10.1055/a-1579-5308. ISSN 0340-6245. 
Table 1 The effect of ChAdOx1 nCoV-19 Vaccine on platelet function

\begin{tabular}{|c|c|c|c|c|}
\hline & Reference interval $^{a}$ & $\begin{array}{l}\text { With } \\
\text { ChAdOx1 nCoV-19 } \\
\text { vaccine }\end{array}$ & $\begin{array}{l}\text { Without } \\
\text { ChAdOx1 nCoV-19 } \\
\text { vaccine }\end{array}$ & $p$-Value \\
\hline \multicolumn{5}{|c|}{ Spontaneous platelet activation (\% positive platelets) } \\
\hline Activated GPIIb-IIIa & - & $20.0 \pm 12.9$ & $18.4 \pm 12.9$ & 0.45 \\
\hline CD63 & - & $3.0 \pm 0.7$ & $2.7 \pm 0.6$ & 0.33 \\
\hline CD62p & - & $16.4 \pm 6.8$ & $16.9 \pm 5.7$ & 0.74 \\
\hline \multicolumn{5}{|c|}{ TRAP-induced platelet activation (\% positive platelets) } \\
\hline Activated GPIIb-IIIa & $87.7-99.5$ & $91.2 \pm 6.3$ & $92.3 \pm 5.1$ & 0.61 \\
\hline CD63 & 29.4-90.6 & $73.8 \pm 12.6$ & $73.9 \pm 12.9$ & 0.97 \\
\hline CD62p & $93.2-99.7$ & $97.1 \pm 2.4$ & $96.7 \pm 2.8$ & 0.52 \\
\hline \multicolumn{5}{|c|}{ Collagen-related peptide-induced platelet activation (\% positive platelets) } \\
\hline Activated GPIIb-IIla & $67.4-99.6$ & $77.3 \pm 10.5$ & $78.7 \pm 9.0$ & 0.21 \\
\hline CD63 & $30.7-89.9$ & $42.4 \pm 11.4$ & $41.5 \pm 9.8$ & 0.39 \\
\hline CD62p & $76.6-99.5$ & $81.4 \pm 10.4$ & $81.9 \pm 8.2$ & 0.74 \\
\hline \multicolumn{5}{|c|}{ ADP-induced platelet activation, (\% positive platelets) } \\
\hline Activated GPIIb-IIla & $90.9-99.5$ & $96.3 \pm 2.1$ & $97.0 \pm 1.6$ & 0.05 \\
\hline CD63 & $20.6-72.7$ & $56.9 \pm 12.9$ & $59.5 \pm 11.7$ & 0.17 \\
\hline CD62p & $88.2-99.1$ & $96.9 \pm 1.5$ & $97.1 \pm 1.4$ & 0.42 \\
\hline \multicolumn{5}{|l|}{ Platelet aggregation, whole blood ( $\mathrm{AU} \times \mathrm{min})$} \\
\hline Spontaneous platelet aggregation & & $109 \pm 54.2$ & $153 \pm 53.6$ & $<0.01$ \\
\hline TRAP-induced platelet aggregation & $401-1,030$ & $971 \pm 210$ & $1010 \pm 292$ & 0.47 \\
\hline ADP-induced platelet aggregation & $647-1,110$ & $508 \pm 128$ & $532 \pm 240$ & 0.70 \\
\hline Collagen-induced platelet aggregation & $452-892$ & $781 \pm 139$ & $802 \pm 175$ & 0.62 \\
\hline \multicolumn{5}{|c|}{ Platelet aggregation, isolated platelets (\% aggregation) } \\
\hline Spontaneous platelet aggregation & $0-3$ & $0.7 \pm 0.5$ & $0.7 \pm 1.2$ & 1.00 \\
\hline TRAP-induced platelet aggregation & $40-70$ & $45 \pm 10$ & $44 \pm 9$ & 0.90 \\
\hline ADP-induced platelet aggregation & $38-78$ & $47 \pm 14$ & $43 \pm 12$ & 0.07 \\
\hline Collagen-induced platelet aggregation & $60-76$ & $66 \pm 3$ & $64 \pm 3$ & 0.14 \\
\hline \multicolumn{5}{|l|}{ Platelet Function Analyser-200 (s) } \\
\hline Collagen/ADP & $67-127$ & $92 \pm 16$ & $93 \pm 15$ & 0.60 \\
\hline
\end{tabular}

Abbreviations: ADP, adenosine diphosphate; AU, arbitrary units; GP, glycoprotein; TRAP, thrombin receptor activating peptide. Note: The results are presented as means and standard deviations, and $p$-values are based on paired $t$-test.

${ }^{\mathrm{a}}$ Reference intervals are locally established.

dense granule, respectively) and the activation of the fibrinogen receptor GPIIb/IIIa (PAC-1 binding) in samples with versus without vaccine, demonstrating that the vaccine did not introduce spontaneous platelet activation. Neither did the vaccine reduce platelet activation capacity following addition of agonists. In accordance, platelet aggregation response to all agonists was unaltered by the vaccine in both whole blood using Multiplate and in the flow cytometric platelet aggregation assay, where platelet aggregation of isolated platelets is determined independent of platelet count. Moreover, the PFA-200 showed no difference in closure times in samples with vaccine compared with samples without vaccine, demonstrating unaltered global hemostasis reflecting platelet adhesion, activation, and aggregation in whole blood. The vaccine had no effect on the expression of platelet receptors (data not shown). In the drug-induced thrombocytopenia assay, the addition of vaccine with or without addition of human PF4 did not result in binding of autoantibodies to platelets.

Overall, the ChAdOx1 nCoV-19 vaccine from OxfordAstraZeneca did not alter platelet function except from a lower spontaneous platelet aggregation response in whole blood. The reduced spontaneous platelet aggregation does not seem to be directed through direct inhibition of platelet activation as platelet activation capacity was unaltered by vaccine administration. Thus, the finding may be either an artifact or caused by other blood cells, as an effect was only detected in whole blood and not observed when evaluating 
platelet aggregation on isolated platelets. Nevertheless, reduced spontaneous platelet aggregation would not result in thrombosis formation, which is the main finding in VITT, but instead a bleeding phenotype.

We added vaccine in a concentration corresponding to administration of a full vaccine dose directly into the blood stream. This does not exclude that a transiently higher concentration of vaccine is present at the site of injection in vivo leading to platelet activation locally. However, this is not likely the cause of thrombus formation in vivo as it would be a shortlived phenomenon as the vaccine distribution is a rapid firstorder reaction. In addition, in our experiments, the vaccine was added to the samples as the final step and thus to some extent imitates the direct administration into the blood stream.

Overall, the drug-induced thrombocytopenia test results were in congruence with the control experiments on healthy donors' platelets in a recent case series. ${ }^{2}$ Greinacher et al reported only a limited effect of high concentrations of ChAdOx1 nCoV-19 on washed platelet reactivity, which they attributed to an in vitro artifact. ${ }^{2}$ Thus, the vaccine does not lead to platelet reactivity of antibodies that are present in the blood at the administration. However, it does not exclude that the vaccine induces production of platelet activating antibodies in vivo.

In conclusion, addition of ChAdOx $1 \mathrm{nCoV}-19$ vaccine from Oxford-AstraZeneca to citrate-anticoagulated whole blood in vitro does not lead to spontaneous platelet activation or platelet aggregation, nor does the vaccine directly inhibit platelet activation and aggregation responses, or cause binding of autologous antibodies to platelets. Thus, intravasal injection of the vaccine seems to be an unlikely direct trigger for the VITT syndrome. Future studies providing in vivo data from an animal model with intravascular ChAdOx1 nCoV-19 administration are needed to conclude that intravasal injection is not inducing VITT.
Ethical Approval

This study was approved by the Scientific Ethical Committee of Southern Denmark (record no. S-20210043C), and the Region of Southern Denmark (record no. 21/21943).

Conflict of Interest

None declared.

\section{References}

1 Schultz NH, Sørvoll IH, Michelsen AE, et al. Thrombosis and thrombocytopenia after ChAdOx1 $\mathrm{nCoV}-19$ vaccination. $\mathrm{N}$ Engl J Med 2021;384(22):2124-2130

2 Greinacher A, Thiele T, Warkentin TE, Weisser K, Kyrle PA, Eichinger S. Thrombotic thrombocytopenia after ChAdOx1 nCov-19 vaccination. N Engl J Med 2021;384(22):2092-2101

3 Stone D, Liu Y, Shayakhmetov D, Li ZY, Ni S, Lieber A. Adenovirusplatelet interaction in blood causes virus sequestration to the reticuloendothelial system of the liver. J Virol 2007;81(09): 4866-4871

4 Nicolai L, Leunig A, Pekayvaz K, et al. Thrombocytopenia and splenic platelet directed immune responses after intravenous ChAdOx1 nCov-19 administration. bioRxiv 2021. Doi: 10.1101/2021.1106.1129.450356

5 Just Vinholt P, Højrup Knudsen G, Sperling S, Frederiksen H, Nielsen C. Platelet function tests predict bleeding in patients with acute myeloid leukemia and thrombocytopenia. Am J Hematol 2019;94(08):891-901

6 Arnold DM, Curtis BR, Bakchoul TPlatelet Immunology Scientific Subcommittee of the International Society on Thrombosis and Hemostasis. Recommendations for standardization of laboratory testing for drug-induced immune thrombocytopenia: communication from the SSC of the ISTH. J Thromb Haemost 2015;13(04): 676-678

7 Vinholt PJ, Alnor A, Nybo M, Hvas AM. Prediction of bleeding and prophylactic platelet transfusions in cancer patients with thrombocytopenia. Platelets 2016;27(06):547-554 